IJMMS 28:10 (2001) 609-619

PII. S0161171201006263

http://ijmms.hindawi.com

(C) Hindawi Publishing Corp.

\title{
ERGODIC TYPE SOLUTIONS OF DIFFERENTIAL EQUATIONS WITH PIECEWISE CONSTANT ARGUMENTS
}

\author{
ANA ISABEL ALONSO and JIALIN HONG
}

(Received 27 November 2000)

\begin{abstract}
We summarize the conditions discovered for the existence of new ergodic type solutions (asymptotically almost periodic, pseudo almost periodic, ....) of differential equations with piecewise constant arguments. Their existence is characterized by introducing a new tool, the ergodic sequences.
\end{abstract}

2000 Mathematics Subject Classification. 39-02, 34K14, 34D09.

1. Introduction. Equations with piecewise continuous arguments (EPCA) arise in an attempt to extend the theory of functional differential equations with continuous arguments to differential equations with discontinuous arguments. This task is of considerable applied interest since EPCA include, as particular cases, impulsive and loaded equations of control theory and are similar to those found in some biomedical models. The study of such equations has been initiated by Wiener [26], Cooke and Wiener [13], and Shah and Wiener [25] in the 80's; and in each of the areas (existence, asymptotic behavior, periodic and oscillating solutions, approximation, application to control theory, biomedical models and problems of mathematical physics) there appears to be ample opportunity for extending the known results. For specific references (see $[1,2,11,13,14,15,23,25,26,27,28,29,30]$ ).

The theory of almost periodic functions was started between 1923 and 1925 by $\mathrm{H}$. Bohr, and his work quickly attracted the interest of a number of researchers who made many important contributions to its development (see [8, 10, 16, 17, 18, 21, 24, $30,31]$ ). Recently some new ergodic type functions have been introduced and applied to differential equations (see [3, 4, 9, 33]).

The aim of this paper is to bring together both concepts; we prove the existence of ergodic type solutions for some classes of differential equations with piecewise constant argument.

1.1. Ergodic type functions. In this section we introduce the definitions of the different classes of ergodic type functions we are going to deal with.

Let $\mathscr{C}\left(\mathbb{R}, \mathbb{R}^{d}\right)$ (resp., $\mathscr{C}\left(\mathbb{R} \times \Omega, \mathbb{R}^{d}\right)$, where $\Omega \subset \mathbb{R}^{d}$ ) denote the Banach space of bounded continuous functions $\varphi(t)$ (resp., $\varphi(t, x)$ ) from $\mathbb{R}$ (resp., $\mathbb{R} \times \Omega$ ) to $\mathbb{R}^{d}$, endowed with the norm $\|\varphi\|=\sup _{t \in \mathbb{R}}|\varphi(t)|$ (resp., $\|\varphi\|=\sup _{t \in \mathbb{R}, x \in \Omega}|\varphi(t, x)|$ ).

Definition 1.1. A function $f \in \mathscr{C}\left(\mathbb{R}^{+}, \mathbb{R}^{d}\right)$ (resp., $\mathscr{C}\left(\mathbb{R}^{+} \times \Omega, \mathbb{R}^{d}\right)$ ) is called asymptotically almost periodic if $f=\left.f_{1}\right|_{\mathbb{R}^{+}}+f_{0}$ where $f_{1}$ is an almost periodic function and $f_{0} \in \mathscr{A} \mathscr{A} \mathscr{P}_{0}$, where $\mathscr{A} \mathscr{A} \mathscr{P}_{0}\left(\mathbb{R}^{+}, \mathbb{R}^{d}\right)=\left\{f \in \mathscr{C}\left(\mathbb{R}^{+}, \mathbb{R}^{d}\right): \lim _{t \rightarrow+\infty} f(t)=0\right\}$ and, 
respectively, $\mathscr{A} \mathscr{A} \mathscr{P}_{0}\left(\mathbb{R}^{+} \times \Omega, \mathbb{R}^{d}\right)=\left\{\lim _{t \rightarrow+\infty} f(t, x)=0\right.$ uniformly for $x$ in compact subsets of $\Omega$ \}.

Let $\mathscr{A} \mathscr{A} \mathscr{P}\left(\mathbb{R}^{+}, \mathbb{R}^{d}\right)\left(\right.$ resp., $\left.\mathscr{A} \mathscr{A} \mathscr{P}_{0}\left(\mathbb{R}^{+} \times \Omega, \mathbb{R}^{d}\right)\right)$ denote the set of all such functions.

For $\varphi \in \mathscr{C}\left(\mathbb{R}, \mathbb{R}^{d}\right)\left(\right.$ resp., $\left.\mathscr{C}\left(\mathbb{R} \times \Omega, \mathbb{R}^{d}\right)\right)$ we define

$$
m(|\varphi|)=\lim _{T \rightarrow+\infty} \frac{1}{2 T} \int_{-T}^{T}|\varphi(t)| d t, \quad\left(\text { resp., } m(|\varphi|)=\lim _{T \rightarrow+\infty} \frac{1}{2 T} \int_{-T}^{T}|\varphi(t, x)| d t\right) .
$$

Definition 1.2 (see [3, 4, 9, 32, 33]). A function $f \in \mathscr{C}\left(\mathbb{R}, \mathbb{R}^{d}\right)$ (resp., $\mathscr{C}\left(\mathbb{R} \times \Omega, \mathbb{R}^{d}\right)$ ) is called pseudo almost periodic if $f=f_{1}+f_{0}$, where $f_{1}$ is almost periodic in $t \in \mathbb{R}$ (almost periodic in $t \in \mathbb{R}$, uniformly in $x \in \Omega$ ) and $f_{0} \in \mathscr{P} \mathscr{A} \mathscr{P}_{0}\left(\mathbb{R}, \mathbb{R}^{d}\right)$ (resp., $\mathscr{P} \mathscr{A}_{\mathscr{P}_{0}}(\mathbb{R} \times$ $\left.\Omega, \mathbb{R}^{d}\right)$ ), where

$$
\begin{gathered}
\mathscr{P} \mathscr{A} \mathscr{P}_{0}\left(\mathbb{R}, \mathbb{R}^{d}\right)=\left\{\varphi \in \mathscr{C}\left(\mathbb{R}, \mathbb{R}^{d}\right): m(|\varphi|)=0\right\}, \\
\mathscr{P} \mathscr{A} \mathscr{P}_{0}\left(\mathbb{R} \times \Omega, \mathbb{R}^{d}\right)=\left\{\varphi \in \mathscr{C}\left(\mathbb{R} \times \Omega, \mathbb{R}^{d}\right): m(|\varphi|)=0 \text { uniformly in } x \in \Omega\right\} .
\end{gathered}
$$

We denote by $\mathscr{P} \mathscr{A} \mathscr{P}\left(\mathbb{R}, \mathbb{R}^{d}\right)$ (resp., $\mathscr{P} \mathscr{A} \mathscr{P}\left(\mathbb{R} \times \Omega, \mathbb{R}^{d}\right)$ ) the set of all such functions.

DEFINITION 1.3. A Lebesgue measurable function $f$ from $\mathbb{R}$ to $\mathbb{R}^{d}$ (resp., from $\mathbb{R} \times \Omega$ to $\mathbb{R}^{d}$ ) is called generalized pseudo almost periodic (see [3, 4]) if $f=f_{1}+f_{0}$, where $f_{1}$ is almost periodic in $t \in \mathbb{R}$ (almost periodic in $t \in \mathbb{R}$, uniformly in $x \in \Omega$ ), and $f_{0} \in \widetilde{\mathscr{P}} \mathscr{A} \mathscr{P}_{0}\left(\mathbb{R}, \mathbb{R}^{d}\right)\left(\right.$ resp., $\left.\widetilde{\mathscr{P}} \mathscr{A} \mathscr{P}_{0}\left(\mathbb{R} \times \Omega, \mathbb{R}^{d}\right)\right)$, where

$$
\begin{gathered}
\widetilde{\mathscr{P}} \mathscr{A}_{\mathscr{P}_{0}}\left(\mathbb{R}, \mathbb{R}^{d}\right)=\left\{\varphi: \mathbb{R} \rightarrow \mathbb{R}^{d} \text { Lebesgue measurable and } m(|\varphi|)=0\right\}, \\
\widetilde{\mathscr{P}} \mathscr{A} \mathscr{P}_{0}\left(\mathbb{R} \times \Omega, \mathbb{R}^{d}\right)=\left\{\varphi: \mathbb{R} \times \Omega \longrightarrow \mathbb{R}^{d} / \varphi(\cdot, x) \in \widetilde{\mathscr{P}} \mathscr{A}_{\mathscr{P}_{0}}\left(\mathbb{R}, \mathbb{R}^{d}\right) \forall x \in \Omega, m(|\varphi|)=0\right\} .
\end{gathered}
$$

We denote by $\widetilde{\mathscr{P}} \mathscr{A} \mathscr{P}\left(\mathbb{R}, \mathbb{R}^{d}\right)\left(\right.$ resp., $\left.\widetilde{\mathscr{P}} \mathscr{A} \mathscr{P}\left(\mathbb{R} \times \Omega, \mathbb{R}^{d}\right)\right)$ the set of all such functions.

In Definitions 1.2 and 1.3 , the functions $f_{1}$ and $f_{2}$ are called the almost periodic component and the ergodic perturbation, respectively, of the function $f$; and they are uniquely determined by $f$ (see [4, page 1143]).

REMARK 1.4. There exist generalized pseudo almost periodic functions whose ergodic component is not bounded. In Ait Dads, Ezzinbi, and Arino [4] it can be found an example of this situation; a long and interesting proof (pages 1143-1146) shows that the function $\varphi(t)=t \mid \sin \pi t t^{t^{N}}$ for $N>6$, is unbounded and $\varphi \in \widetilde{\mathscr{P}} \mathscr{A}_{\mathscr{P}_{0}}(\mathbb{R}, \mathbb{R})$.

We propose the function

$$
f(t)= \begin{cases}2 k\left(t-2^{k^{2}}+1\right), & \text { if } 2^{k^{2}}-1 \leq t \leq 2^{k^{2}}-\frac{1}{2}, \\ -2 k\left(t-2^{k^{2}}\right), & \text { if } 2^{k^{2}}-\frac{1}{2} \leq t \leq 2^{k^{2}}, \\ 0, & \text { otherwise, }\end{cases}
$$

where $k \in \mathbb{Z}^{+}$. It is short to prove that $f \in \widetilde{\mathscr{P}} \mathscr{A}_{\mathscr{P}_{0}}(\mathbb{R}, \mathbb{R})$ and is unbounded. 
Let $\mathscr{L}\left(\mathbb{R}, \mathbb{R}^{d}\right)$ (resp., $\mathscr{L}\left(\mathbb{R} \times \Omega, \mathbb{R}^{d}\right)$ ) denote the space of all Lebesgue measurable and bounded functions $f(t)$ (resp., $f(t, x)$ ) from $\mathbb{R}$ (resp., $\mathbb{R} \times \Omega$ ) to $\mathbb{R}^{d}$.

DEFINITION 1.5. A function $f \in \mathscr{L}\left(\mathbb{R}, \mathbb{R}^{d}\right)$ is said to be ergodic (see [19]) if the limit $\lim _{T \rightarrow+\infty}(1 / 2 T) \int_{-T}^{T} f(t) d t=M(f)$ exists. Analogously, we say that a function $f \in$ $\mathscr{L}\left(\mathbb{R} \times \Omega, \mathbb{R}^{d}\right)$ is ergodic if for every compact subset $K \subset \Omega$, the $\operatorname{limit}_{\lim _{T \rightarrow+\infty}}(1 / 2 T) \times$ $\int_{-T}^{T} f(t, x) d t=M(f, x)$ exists uniformly for $x \in K$.

We will write $\mathscr{E}\left(\mathbb{R}, \mathbb{R}^{d}\right)$ and $\mathscr{E}\left(\mathbb{R} \times \Omega, \mathbb{R}^{d}\right)$ for the sets of ergodic functions defined on $\mathbb{R}$ and $\mathbb{R} \times \Omega$, respectively.

REMARK 1.6. As it is well known, there are uniformly continuous bounded functions on $\mathbb{R}$ which are not ergodic. For example, the function

$$
f(t)= \begin{cases}1-t^{2}, & \text { if }|t|<1 \\ \sin \left(\log \left(\frac{1}{t^{2}}\right)\right), & \text { if }|t| \geq 1\end{cases}
$$

is uniformly continuous in $\mathbb{R}$, but $f$ is not ergodic.

The relations between the functions defined above can be expressed as follows:

$$
\begin{gathered}
\mathscr{P} \mathscr{A} \mathscr{P}\left(\mathbb{R}, \mathbb{R}^{d}\right) \subset \widetilde{\mathscr{P}} \mathscr{A} \mathscr{P}\left(\mathbb{R}, \mathbb{R}^{d}\right), \\
\mathscr{P}\left(\mathbb{R}, \mathbb{R}^{d}\right) \subset \mathscr{\mathscr { P }}\left(\mathbb{R}, \mathbb{R}^{d}\right) \subset \mathscr{A} \mathscr{P}\left(\mathbb{R}, \mathbb{R}^{d}\right) \subset \mathscr{P} \mathscr{A} \mathscr{P}\left(\mathbb{R}, \mathbb{R}^{d}\right) \subset \mathscr{E}\left(\mathbb{R}, \mathbb{R}^{d}\right),
\end{gathered}
$$

where $\mathscr{P}, \mathscr{2} \mathscr{P}$, and $\mathscr{A} \mathscr{P}$ refer to periodic, quasi periodic and almost periodic functions, respectively.

1.2. Ergodic type sequences. Here we introduce and discuss some class of ergodic sequences which are the main tools in the proofs of our results.

Definition 1.7 (see $[6,7,20]$ ). (1) A sequence $x: \mathbb{Z} \rightarrow \mathbb{R}^{d}$ is said to be a $\tilde{\mathscr{P}} \mathscr{A} \mathscr{P}_{0}$ (resp., $\mathscr{P} \mathscr{A} \mathscr{P}_{0}$ ) sequence if it satisfies

$$
\lim _{n \rightarrow+\infty} \frac{1}{2 n} \sum_{k=-n}^{n}|x(k)|=0, \quad\left(\lim _{n \rightarrow+\infty} \frac{1}{2 n} \sum_{k=-n}^{n}|x(k)|=0 \text { and it is bounded }\right) .
$$

(2) A sequence $x: \mathbb{Z} \rightarrow \mathbb{R}^{d}$ is said to be a $\widetilde{P} \mathscr{A} \mathscr{P}($ resp., $\mathscr{P} \mathscr{A} \mathscr{P})$ sequence if $x=x_{1}+x_{0}$, where $x_{1}$ is an almost periodic sequence (see $\left.[5,17,30]\right)$ and $x_{0}$ is a $\widetilde{P}_{\mathscr{A}} \mathscr{P}_{0}\left(\right.$ resp., $\left.\mathscr{P} \mathscr{A} \mathscr{P}_{0}\right)$ sequence.

REMARK 1.8. Notice that

(1) a sequence vanishing at infinity is a $\mathscr{P} \mathscr{A} \mathscr{P}_{0}$ sequence;

(2) the sequence $\{x(n)\}_{n \in \mathbb{Z}}$ defined by $x(n)=1$ if $n=2^{k} ; x(n)=0$ otherwise, is an example of a $\mathscr{P} \mathscr{A} \mathscr{P}_{0}$ sequence which is not vanishing at infinity;

(3) for $k \in \mathbb{Z}^{+}$the sequence $\{x(n)\}_{n \in \mathbb{Z}}$, defined by $x(n)=k$ if $n=2^{k^{2}} ; x(n)=0$ otherwise, is an example of an unbounded $\mathscr{P} \mathscr{A} \mathscr{P}_{0}$ sequence.

DEFINITION 1.9. A bounded sequence $\{x(n)\}_{n \in \mathbb{Z}}$ is said to be ergodic (see [19]) if the $\operatorname{limit}_{\lim _{n \rightarrow+\infty}}(1 / 2 n) \sum_{k=-n}^{n} x(k)$ exists. We denote by $\mathscr{E}(\mathbb{Z})$ the set of all such sequences. 
The following propositions and examples show the relations between almost periodic type functions and sequences.

Proposition 1.10. The following statements hold:

(1) (see $[6,7])$. If $\{x(n)\}_{n \in \mathbb{Z}}$ is a $\mathscr{P} \mathscr{A} \mathscr{P}_{0}$ (resp., $\widetilde{P} \mathscr{A} \mathscr{P}_{0}, \mathscr{P} \mathscr{A} \mathscr{P}$, or $\left.\widetilde{\mathscr{P}} \mathscr{A} \mathscr{P}\right)$ sequence, then there exists a function $f \in \mathscr{P} \mathscr{A} \mathscr{P}_{0}\left(\mathbb{R}, \mathbb{R}^{d}\right)\left(\right.$ resp., $\widetilde{P} \mathscr{A} \mathscr{P}_{0}\left(\mathbb{R}, \mathbb{R}^{d}\right), \mathscr{P} \mathscr{A} \mathscr{P}\left(\mathbb{R}, \mathbb{R}^{d}\right)$, or $\widetilde{\mathscr{P}} \mathscr{A} \mathscr{P}$ $\left.\left(\mathbb{R}, \mathbb{R}^{d}\right)\right)$ such that $f(n)=x(n), n \in \mathbb{Z}$.

(2) (see [19]). If $\{x(n)\}_{n \in \mathbb{Z}} \in \mathscr{E}(\mathbb{Z})$, then there exists an ergodic function $f \in \mathscr{E}\left(\mathbb{R}, \mathbb{R}^{d}\right)$ such that $f(n)=x(n)$ for every $n \in \mathbb{Z}$.

Observe that if we have an almost periodic sequence, the converse of the proposition above is also true (see [17]). Although, if we deal with one of the ergodic type sequences specified in the statement of the proposition, the converse does not work, as we show in the following counterexamples.

EXAMPLE 1.11 (see [7]). The function

$$
f(t)= \begin{cases}2^{|k|}(t-k)+1, & \text { if } t \in\left[k-2^{-|k|}, k\right), \\ -2^{|k|}(t-k)+1, & \text { if } t \in\left[k, k+2^{-|k|}\right] \\ 0, & \text { otherwise, }\end{cases}
$$

where $k \neq 0$, is in $\mathscr{P} \mathscr{A} \mathscr{P}_{0}(\mathbb{R}, \mathbb{R})$. But $f(k)=1$ for every $k \in \mathbb{Z}-\{0\}$, so the sequence $\{f(k)\}_{k \in \mathbb{Z}}$ is not a $\mathscr{P} \mathscr{A} \mathscr{P}_{0}$ sequence.

EXAMPLE 1.12 (see [19]). Consider the sequence $\{x(j)\}_{j \in \mathbb{Z}}$ defined by $x(0)=x(1)=$ 0 , for each $k \geq 0$

$$
x(j)= \begin{cases}3, & \text { if } 2^{2^{k}}+1 \leq j \leq 2^{2 k+1}, \\ 0, & \text { if } 2^{2 k+1} \leq j \leq 2^{2 k+2}\end{cases}
$$

and $x(-j)=x(j)$ for every $j \geq 1$. This sequence is not in $\mathscr{E}(\mathbb{Z})$. Nevertheless, the function $f$, defined as

$$
f(t)= \begin{cases}x(n)\left(1-2^{(n+1)}(t-n)\right), & \text { if } t \in\left[n, n+2^{-(n+1)}\right], \\ 0, & \text { if } t \in\left(n+2^{-(n+1)}, n+1-2^{-(n+2)}\right), \\ x(n+1)\left(1+2^{(n+2)}(t-(n+1))\right), & \text { if } t \in\left[n+1-2^{-(n+2)}, n+1\right],\end{cases}
$$

satisfies that $f(n)=x(n)$ for every $n \in \mathbb{Z}$, and is ergodic.

2. Ergodic type solutions via ergodic type sequences. Meisters, in [21], showed that the existence of almost periodic solutions of ordinary differential equations is equivalent to the fact that the restriction of a bounded solution to some discrete subgroup of reals is almost periodic. This is improved by Opial [22] (also see Fink [17, pages 164-169]).

It is natural to wonder if there are similar results on the existence of almost periodic, asymptotically almost periodic, pseudo almost periodic and ergodic solutions of EPCA. 
In this part we give an affirmative answer to this question under similar conditions to those of Meisters, Opial, and Fink.

2.1. For EPCA of retarded type. Consider the differential equation with piecewise constant argument

$$
\frac{d x}{d t}=f(t, x(t), x([t]), x([t-1]), \ldots, x([t-k])), \quad t \in \mathbb{R},
$$

where $k$ is a positive integer, $f \in C\left(\mathbb{R} \times \Omega\right.$, $\left.\mathbb{R}^{d}\right)$, and [ $\left.\cdot\right]$ denotes the greatest integer function. A function $x: \mathbb{R} \rightarrow \mathbb{R}^{d}$ is called a solution of (2.1) if the following conditions are satisfied:

(1) $x$ is continuous in $\mathbb{R}$,

(2) the derivative $x^{\prime}(t)$ of $x(t)$ exists everywhere, with possible exception of the points $[t]$, where one-sided derivatives exist,

(3) equation (2.1) is satisfied on each interval $[n, n+1)$ with integral end-points.

Now we present our main results for this equation.

THEOREM 2.1 (see [6]). Let $f \in \mathscr{A} \mathscr{P}\left(\mathbb{R} \times \Omega_{0}\right)$ in (2.1) for a compact subset $\Omega_{0} \subset \Omega$. If all the equations

$$
\frac{d x}{d t}=g(t, x(t), x([t]), x([t-1]), \ldots, x([t-k])), \quad t \in \mathbb{R},
$$

with $g$ in the hull of $f$ (see [17] for the definition), have unique solutions to initial value problems, where the initial value condition is $x(j)=x_{j}, j=0,-1,-2, \ldots,-k$, and $\varphi(t)$ is a solution of (2.1) with $\varphi(\mathbb{R})^{k+2} \subset \Omega_{0}$, then $\varphi \in \mathscr{A} \mathscr{P}(\mathbb{R})$ if and only if $\{\varphi(n)\}_{n \in \mathbb{Z}} \in \mathscr{A} \mathscr{P}(\mathbb{Z})$.

THEOREM 2.2 (see [6]). Let $f \in \mathscr{P} \mathscr{A} \mathscr{P}_{0}\left(\mathbb{R} \times \Omega\right.$ ) (resp., $\mathscr{A} \mathscr{A} \mathscr{P}_{0}\left(\mathbb{R}^{+} \times \Omega\right)$ ) in (2.1) satisfying a Lipschitz condition on $\Omega$. If $\varphi$ is a solution of (2.1) with $\varphi(\mathbb{R})^{k+2} \subset \Omega$, then $\varphi \in$ $\mathscr{P} \mathscr{A} \mathscr{P}_{0}(\mathbb{R})\left(\right.$ resp., $\left.\mathscr{A} A \mathscr{P}_{0}\left(\mathbb{R}^{+}\right)\right)$if and only if $\{\varphi(n)\}_{n \in \mathbb{Z}} \in \mathscr{P} \mathscr{A} \mathscr{P}_{0}(\mathbb{Z})\left(\right.$ resp., $\mathscr{A}_{A} \mathscr{P}_{0}\left(\mathbb{Z}^{+}\right)$).

THEOREM 2.3 (see [6]). Let $f \in \mathscr{P} \mathscr{A} \mathscr{P}\left(r \times \Omega_{0}\right)$ (resp., $\mathscr{A} \mathscr{A} \mathscr{P}\left(\mathbb{R}^{+} \times \Omega_{0}\right)$ ) in (2.1) for a compact subset $\Omega_{0} \subset \Omega$, and suppose $f$ and its almost periodic component, $f_{1}$, satisfy a Lipschitz condition on $\Omega_{0}$ with Lipschitz constant L. If $\varphi(t)$ is a solution of (2.1) with $\varphi(\mathbb{R})^{k+2} \subset \Omega_{0}$, then $\varphi \in \mathscr{P} \mathscr{A P}(\mathbb{R})\left(\right.$ resp., $\mathscr{A} \mathscr{A} \mathscr{P}\left(\mathbb{R}^{+}\right)$) if and only if $\{\varphi(n)\}_{n \in \mathbb{Z}} \in \mathscr{P} \mathscr{A} \mathscr{P}(\mathbb{Z})$ (resp., $\mathscr{A} \mathscr{A} \mathscr{P}\left(\mathbb{Z}^{+}\right)$).

As an example of how do these theorems apply, we examine the following equation with piecewise constant argument.

EXAMPLE 2.4 (see [6]). Consider the equation

$$
\frac{d x}{d t}=x(t)(a(t)-b(t) x([t]))
$$

where $a(t)$ and $b(t)$ are positive and continuous bounded functions on $\mathbb{R}^{+}$, and satisfy $\int_{n}^{n+1} a(t) d t=\int_{n}^{n+1} b(t) d t$ for every $n \in \mathbb{Z}^{+}$. The above equation is analogous to the 
famous logistic differential equation, but $t$ in one argument has been replaced by $[t]$. We investigate the existence of almost periodic type solutions of this equation.

It follows from

$$
x(n+1)=x(n) e^{\int_{n}^{n+1} a(t) d t} e^{-x(n) \int_{n}^{n+1} b(t) d t}, \quad \text { for } n \in \mathbb{Z}^{+},
$$

that if we take $x(0)=1$, then for every $n \in \mathbb{Z}^{+}$we get $x(n)=1$. According with Theorems 2.1 and 2.3, it is concluded that the equation has a solution $x \in \mathscr{A} \mathscr{P}\left(\mathbb{R}^{+}\right)$ (resp., $x \in \mathscr{P} \mathscr{A} \mathscr{P}\left(\mathbb{R}^{+}\right)$) with $x(0)=1$ if the functions $a, b \in \mathscr{A} \mathscr{P}\left(\mathbb{R}^{+}\right)$(resp., $a, b \in$ $\left.\mathscr{P} \mathscr{A} \mathscr{P}\left(\mathbb{R}^{+}\right)\right)$. As a result, the equation has solutions that display complicated dynamics even if $a(t)=b(t)=$ constant (Carvalho and Cooke's equation, see [12]).

2.2. For ordinary differential equations. In order to study ergodic solutions of differential equations via ergodic sequences, we introduce the concept of a $\mathscr{T}$-ergodic function.

Let $\mathscr{T}$ be the translator operator $\mathscr{T}:[-1,0] \rightarrow[0,1], \tau \mapsto 1+\tau$. We denote by $\mathscr{T}^{k}$ the composition operator, $\mathscr{T}^{0}(\tau)=\tau$ and $\mathscr{T}^{-1}(\tau)=\tau-1$. A function $f \in \mathscr{L}\left(\mathbb{R} \times \Omega, \mathbb{R}^{d}\right)$ is called $\mathscr{T}$-ergodic if for each compact subset $K$ of $\Omega$, the $\operatorname{limit}_{n \rightarrow \infty}(1 / 2 n) \times$ $\sum_{k=-n}^{n} f\left(\mathscr{T}^{k}(\tau), x\right)$ exists in Lebesgue measure on $[-1,0]$, uniformly for $x \in K$.

The property of $\mathscr{T}$-ergodicity implies ergodicity but the converse is not true.

Now consider the ordinary differential equation

$$
x^{\prime}(t)=f(t, x(t)), \quad t \in \mathbb{R} .
$$

We have the following results.

THEOREM 2.5 (see [19]). Suppose that for a function $f \in \mathscr{L}\left(\mathbb{R} \times \Omega, \mathbb{R}^{d}\right)$ and for a solution $x$ of (2.5) the composed function $f(t, x(t))$ is $\mathscr{T}$-ergodic. Then $x \in \mathscr{E}\left(\mathbb{R}, \mathbb{R}^{d}\right)$ if and only if the sequence $\{x(n)\}_{n \in \mathbb{Z}} \in \mathscr{E}(\mathbb{Z})$.

THEOREM 2.6 (see [19]). Let $f \in \mathscr{L}\left(\mathbb{R} \times \Omega, \mathbb{R}^{d}\right)$ satisfy the Lipschitz condition

$$
\left|f\left(t, y_{1}\right)-f\left(t, y_{2}\right)\right| \leq L(t)\left|y_{1}-y_{2}\right|, \quad y_{1}, y_{2} \in \Omega, t \in \mathbb{R}
$$

where the nonnegative function $L(\cdot) \in \widetilde{\mathscr{P}} \mathscr{A} \mathscr{P}(\mathbb{R}, \mathbb{R})$ and suppose that for at least one point $y \in \Omega,\{f(\cdot, y)\}$ is $\mathcal{T}$-ergodic. Then a solution $x(t)$ of (2.5) is ergodic if and only if the sequence $\{x(n)\}_{n \in \mathbb{Z}}$ is ergodic.

THEOREM 2.7 (see [19]). Let $f(t, x) \in \mathscr{L}\left(\mathbb{R} \times \Omega, \mathbb{R}^{d}\right)$ be uniformly continuous in $t$ for $x$ in compact subsets of $\Omega$ and suppose it satisfies a Lipschitz condition with Lipschitz constant $L>0$. Furthermore, assume $f$ is such that for every Lebesgue measurable set $E \subset \mathbb{R}$, the limit

$$
\lim _{T \rightarrow \infty} \frac{1}{2 T} \int_{[-T, T] \cap E} f(t, y) d t
$$

exists for each $y \in \Omega$. Then, if $x(t)$ is a solution of (2.5), $x \in \mathscr{E}\left(\mathbb{R}, \mathbb{R}^{d}\right)$ if and only if the sequence $\{x(n)\}_{n \in \mathbb{Z}} \in \mathscr{E}(\mathbb{Z})$. 
3. Some EPCA of mixed type. Consider the following initial-value problems posed for the differential equations with piecewise argument

$$
\begin{aligned}
& x^{\prime}(t)=a x(t)+\sum_{i=-N}^{N} a_{i} x([t+i])+f(t), \quad N \geq 2, \\
& x^{\prime}(t)=a x(t)+\sum_{i=-N}^{N} a_{i} x([t+i])+g(t, x(t), x([t])), \quad N \geq 2,
\end{aligned}
$$

where $[\cdot]$ denotes the greatest integer function, $a, a_{i}$ are constants, $f \in \widetilde{\mathscr{P}} \mathscr{A} \mathscr{P}(\mathbb{R}, \mathbb{R})$ (resp., $\mathscr{P} \mathscr{A} \mathscr{P}(\mathbb{R}, \mathbb{R})), g \in \widetilde{\mathscr{P}} \mathscr{A} \mathscr{P}\left(\mathbb{R} \times \mathbb{R}^{2}, \mathbb{R}\right)\left(\right.$ resp., $\left.\mathscr{P} \mathscr{A} \mathscr{P}\left(\mathbb{R} \times \mathbb{R}^{2}, \mathbb{R}\right)\right)$ is bounded, and there exists a constant $\eta>0$ such that

$$
\left|g\left(t, x_{1}, y_{1}\right)-g\left(t, x_{2}, y_{2}\right)\right| \leq \eta\left(\left|x_{1}-x_{2}\right|+\left|y_{1}-y_{2}\right|\right)
$$

for every $\left(t, x_{1}, y_{1}\right),\left(t, x_{2}, y_{2}\right) \in \mathbb{R} \times \mathbb{R}^{2}$; and the initial conditions $x(i)=c_{i},-N \leq i \leq$ $N-1$. In contrast to the situation with general functional differential equations, the fact that these equations contain both retarded and advanced arguments does not pose particular difficulties.

The definition of a solution of these equations is analogous to the one given for EPCA of retarded type, in Section 2.1. Obviously, if $x(t)$ is a solution of (3.1) on $\mathbb{R}$, then for $n \leq t \leq n+1$ we have

$$
x(t)=e^{a(t-n)} c_{n}+\left(e^{a(t-n)}-1\right) \sum_{i=-N}^{N} a^{-1} a_{i} c_{n+i}+\int_{n}^{t} e^{a(t-s)} f(s) d s,
$$

where $x(n+i)=c_{n+i},-N \leq i \leq N$. Therefore, it suffices to know the constants $c_{n}$ in order to determine $x(t)$.

Let

$$
\begin{gathered}
b_{0}=e^{a}+a^{-1} a_{0}\left(e^{a}-1\right), \quad b_{1}=a^{-1} a_{1}\left(e^{a}-1\right)-1, \\
b_{i}=a^{-1} a_{i}\left(e^{a}-1\right), \quad i=-1, \pm 2, \ldots, \pm N, \\
h_{n}=-\int_{n}^{n+1} e^{a(n+1-s)} f(s) d s .
\end{gathered}
$$

Continuity of a solution at a point joining any two consecutive intervals leads to recursion relations for the solution at such points which, by virtue of (3.5), can be written as $\sum_{i=-N}^{N} b_{i} c_{n+i}=h_{n}$. A particular solution of the corresponding homogeneous equation is sought as $c_{n}=\lambda^{n}$ (see [15]), then

$$
\sum_{i=-N}^{N} b_{i} \lambda^{n+i}=0
$$

Our main results are as follows.

THEOREM 3.1 (see [7]). Suppose that all roots of (3.6) are simple (denoted by $\lambda_{1}, \ldots$, $\left.\lambda_{2 N}\right)$ and $\left|\lambda_{i}\right| \neq 1,1 \leq i \leq 2 N$. Then

(1) for any $f \in \widetilde{\mathscr{P}} \mathscr{A P}_{0}(\mathbb{R}, \mathbb{R})\left(\right.$ resp., $\mathscr{P} \mathscr{A} \mathscr{P}_{0}(\mathbb{R}, \mathbb{R})$ or $\widetilde{P} \mathscr{A} \mathscr{P}_{0}(\mathbb{R}, \mathbb{R}) \cap M_{b}(\mathbb{R}, \mathbb{R})$ ), (3.1) 
has a solution $x \in \widetilde{P} \mathscr{A}_{0}(\mathbb{R}, \mathbb{R})$ (resp., $\mathscr{P} \mathscr{A} \mathscr{P}_{0}(\mathbb{R}, \mathbb{R})$ ), besides $x$ is unique if $f \in$ $\mathscr{P} \mathscr{A} \mathscr{P}_{0}(\mathbb{R}, \mathbb{R})$ or $f \in \tilde{\mathscr{P}} \mathscr{A}_{0}(\mathbb{R}, \mathbb{R}) \cap M_{b}(\mathbb{R}, \mathbb{R})$, where

$M_{b}(\mathbb{R}, \mathbb{R})=\{\varphi: \mathbb{R} \rightarrow \mathbb{R}$ is Lebesgue measurable and bounded on $\mathbb{R}\}$

(2) for any $f \in \widetilde{\mathscr{P}} \mathscr{A} \mathscr{P}(\mathbb{R}, \mathbb{R})\left(\right.$ resp., $\mathscr{P} \mathscr{A} \mathscr{P}(\mathbb{R}, \mathbb{R})$ or $\widetilde{\mathscr{P}} \mathscr{A} \mathscr{P}(\mathbb{R}, \mathbb{R}) \cap M_{b}(\mathbb{R}, \mathbb{R})$ ), (3.1) has a solution $x \in \widetilde{\mathscr{P}} \mathscr{A P}(\mathbb{R}, \mathbb{R})$ (resp., $\mathscr{P} \mathscr{A} \mathscr{P}(\mathbb{R}, \mathbb{R})$ ), and $x$ is unique if $f \in \mathscr{P} \mathscr{A} \mathscr{P}(\mathbb{R}, \mathbb{R})$ or $f \in \widetilde{\mathscr{P}} \mathscr{A} \mathscr{P}(\mathbb{R}, \mathbb{R}) \cap M_{b}(\mathbb{R}, \mathbb{R})$.

THEOREM 3.2 (see [7]). Suppose that all roots of (3.6) are simple (denoted by $\lambda_{1}, \ldots$, $\left.\lambda_{2 N}\right)$ and $\left|\lambda_{i}\right| \neq 1,1 \leq i \leq 2 N$. Then there exists $\eta_{*}>0$, such that

(1) when $0 \leq \eta<\eta_{*}$, (3.2) has a unique solution $x \in \mathscr{P} \mathscr{A} \mathscr{P}_{0}(\mathbb{R}, \mathbb{R})$ if $g \in \widetilde{\mathscr{P}} \mathscr{A}_{0}(\mathbb{R} \times$ $\left.\mathbb{R}^{2}, \mathbb{R}\right)$ is bounded and satisfies the Lipschitz condition (3.3);

(2) when $0 \leq \eta<\eta_{*}$, (3.2) has a unique solution $x \in \mathscr{P} \mathscr{A} \mathscr{P}(\mathbb{R}, \mathbb{R})$ if $g \in \widetilde{P} \mathscr{A} \mathscr{P}(\mathbb{R} \times$ $\left.\mathbb{R}^{2}, \mathbb{R}\right)$ is bounded and satisfies the Lipschitz condition (3.3).

4. EPCA of neutral type. Solutions and exponential dichotomy. Consider the nonhomogeneous neutral differential equation with piecewise constant argument

$$
y^{\prime}(t)=A(t) y(t)+B(t) y([t])+A_{0}(t) y(t-[t])+A_{1}(t) y^{\prime}(t-[t])+f(t),
$$

and the nonlinear neutral differential equations of the form

$$
\begin{aligned}
y^{\prime}(t)= & A(t) y(t)+B(t) y([t]) \\
& +A_{0}(t) y(t-[t])+A_{1}(t) y^{\prime}(t-[t])+g(t, y(t), y([t])),
\end{aligned}
$$

where $A, B, A_{0}, A_{1}: \mathbb{R} \rightarrow \mathbb{R}^{d \times d}, f: \mathbb{R} \rightarrow \mathbb{R}^{d}, g: \mathbb{R} \times \mathbb{R}^{d} \rightarrow \mathbb{R}^{d}$ are Lebesgue measurable. Throughout this section, we assume that there exists $\eta>0$ such that

$$
\left|g\left(t, x_{1}, y_{1}\right)-g\left(t, x_{2}, y_{2}\right)\right| \leq \eta\left[\left|x_{1}-x_{2}\right|+\left|y_{1}-y_{2}\right|\right], \quad x_{i}, y_{i} \in \mathbb{R} .
$$

Let $X(t)$ be the fundamental matrix solution of $x^{\prime}(t)=A(t) x(t)$ such that $X(0)=$ Id and $y(t)$ a solution of (4.1). If we set $y_{0}(t)=\left.y(t)\right|_{[0,1]}$ and define

$$
\begin{gathered}
C(n)=X(n+1)\left[X^{-1}(n)+\int_{n}^{n+1} X^{-1}(u) B(u) d u\right], \\
h(n)=X(n+1) \int_{n}^{n+1} X^{-1}(u)\left[A_{0}(u) y_{0}(u-n)+A_{1}(u) y_{0}^{\prime}(u-n)+f(u)\right] d u,
\end{gathered}
$$

then $\{y(n)\}_{n \in \mathbb{Z}}$ satisfies the nonhomogeneous difference equation

$$
y(n+1)=C(n) y(n)+h(n), \quad n \in \mathbb{Z} .
$$

We assume that for each $n \in \mathbb{Z}, C(n)$ is an invertible $d \times d$ matrix.

DefinITION 4.1 (see [20, 27, 31]). (1) Equation (4.5) is said to admit an exponential dichotomy on $\mathbb{Z}$ if there exist positive constants $K \geq 1, \alpha>0$ and a projection $P$ 
$\left(P^{2}=P\right)$ such that

$$
\begin{aligned}
\left|Y(n) P Y^{-1}(m)\right| \leq K e^{-\alpha(n-m)}, & n \geq m, \\
\left|Y(n)(I-P) Y^{-1}(m)\right| \leq K e^{-\alpha(n-m)}, & m \geq n,
\end{aligned}
$$

where $Y(n)$ is the fundamental matrix solution of (4.5) with $Y(0)=$ Id.

(2) The linear differential equation with piecewise constant argument

$$
y^{\prime}(t)=A(t) y(t)+B(t) y([t])
$$

is said to have an exponential dichotomy if the difference equation (4.5) has an exponential dichotomy.

Now we give our main results.

THEOREM 4.2 (see [20]). Suppose that $A(t)$ and $B(t)$ are Lebesgue measurable and bounded, and (4.7) admits an exponential dichotomy. If $A_{0}, A_{1} \in \widetilde{\mathscr{P}} \mathscr{A}_{\mathscr{P}_{0}}\left(\mathbb{R}, \mathbb{R}^{d \times d}\right) \cap M_{b}$, $f \in \widetilde{\mathscr{P}} \mathscr{A}_{\mathscr{P}_{0}}\left(\mathbb{R}, \mathbb{R}^{d}\right) \cap M_{b}$, and $\left|A_{1}\right|=\sup _{t \in \mathbb{R}}\left|A_{1}(t)\right|<1$, then there exists $\beta_{0}>0$ such that when $\left|A_{0}\right|+\left|A_{1}\right|<\beta_{0}$, (4.1) has a unique solution $x \in \mathscr{P} \mathscr{A P}_{0}\left(\mathbb{R}, \mathbb{R}^{d}\right)$, where

$$
M_{b}\left(\mathbb{R}, \mathbb{R}^{d}\right)=\left\{f \mid f: \mathbb{R} \rightarrow \mathbb{R}^{d} \text { is Lebesgue measurable and bounded }\right\}
$$

THEOREM 4.3 (see [20]). Suppose that $A(t)$ and $B(t)$ are Lebesgue measurable and bounded, and (4.7) admits an exponential dichotomy. If $A_{0}, A_{1} \in \widetilde{\mathscr{P}} \mathscr{A}_{\mathscr{P}_{0}}\left(\mathbb{R}, \mathbb{R}^{d \times d}\right) \cap M_{b}$, $\left|A_{1}\right|<1$, and $g \in \widetilde{\mathscr{P}} \mathscr{A}_{0}\left(\mathbb{R} \times \mathbb{R}^{d} \times \mathbb{R}^{d}, \mathbb{R}^{d}\right)$ is bounded and satisfies the Lipschitz condition (4.3), then there exists $\beta_{1}>0$ and $\eta_{1}>0$ such that when $\left|A_{1}\right|+\left|A_{0}\right|<\beta_{1}$ and $0 \leq \eta<\eta_{1}$, (4.2) has a unique solution $y \in \mathscr{P} \mathscr{A} \mathscr{P}_{0}\left(\mathbb{R}, \mathbb{R}^{d}\right)$.

THEOREM 4.4 (see [20]). Suppose that $A(t)$ y $B(t)$ is almost periodic, $A_{1}(t)=A_{0}(t) \equiv$ 0 and (4.7) admits an exponential dichotomy. Then, the following facts hold:

(1) for any $f \in \widetilde{\mathscr{P}} \mathscr{A P}_{0}\left(\mathbb{R}, \mathbb{R}^{d}\right)$, (resp., $\left.\mathscr{P} \mathscr{A}_{\mathscr{P}_{0}}\left(\mathbb{R}, \mathbb{R}^{d}\right)\right)$, (4.1) has a solution $x \in \widetilde{\mathscr{P}} \mathscr{A}_{\mathscr{P}_{0}}$ $\left(\mathbb{R}, \mathbb{R}^{d}\right)\left(\right.$ resp., $\left.\mathscr{P} \mathscr{A} \mathscr{P}_{0}\left(\mathbb{R}, \mathbb{R}^{d}\right)\right)$, besides, $x$ is unique if $f \in \mathscr{P} \mathscr{A} \mathscr{P}_{0}\left(\mathbb{R}, \mathbb{R}^{d}\right)$ or $\widetilde{P} \mathscr{A}_{\mathscr{P}_{0}}$ $\left(\mathbb{R}, \mathbb{R}^{d}\right) \cap M_{b}\left(\mathbb{R}, \mathbb{R}^{d}\right) ;$

(2) for any $f \in \widetilde{\mathscr{P}} \mathscr{A} \mathscr{P}\left(\mathbb{R}, \mathbb{R}^{d}\right)$, (resp., $\left.\mathscr{P} \mathscr{A} \mathscr{P}\left(\mathbb{R}, \mathbb{R}^{d}\right)\right)$, (4.1) has a solution $x \in \widetilde{\mathscr{P}} \mathscr{A} \mathscr{P} \times$ $\left(\mathbb{R}, \mathbb{R}^{d}\right)\left(\right.$ resp., $\left.\mathscr{P} \mathscr{A P}\left(\mathbb{R}, \mathbb{R}^{d}\right)\right)$, besides, $x$ is unique if $f \in \mathscr{P} \mathscr{A} \mathscr{P}\left(\mathbb{R}, \mathbb{R}^{d}\right)$ or $\widetilde{\mathscr{P}} \mathscr{A} \mathscr{P}$ $\left(\mathbb{R}, \mathbb{R}^{d}\right) \cap M_{b}\left(\mathbb{R}, \mathbb{R}^{d}\right)$.

TheOREM 4.5 (see [20]). Suppose that $A(t)$ y $B(t)$ is almost periodic, $A_{1}(t)=A_{0}(t) \equiv$ 0 and (4.7) admits an exponential dichotomy. Then there exists $\eta_{*}>0$ such that the following facts hold

(1) when $0 \leq \eta<\eta_{*}$, (4.2) has a unique solution $y \in \mathscr{P} \mathscr{A} \mathscr{P}_{0}\left(\mathbb{R}, \mathbb{R}^{d}\right)$ if $g \in \widetilde{\mathscr{P}} \mathscr{A} \mathscr{P}_{0}(\mathbb{R} \times$ $\left.\mathbb{R}^{d} \times \mathbb{R}^{d}, \mathbb{R}^{d}\right)$ is bounded and satisfies the Lipschitz condition (4.3);

(2) when $0 \leq \eta<\eta_{*}$, (4.2) has a unique solution $y \in \mathscr{P} A \mathscr{P}\left(\mathbb{R}, \mathbb{R}^{d}\right)$ if $g \in \widetilde{P} A \mathscr{P}(\mathbb{R} \times$ $\left.\mathbb{R}^{d} \times \mathbb{R}^{d}, \mathbb{R}^{d}\right)$ is bounded and satisfies the Lipschitz condition (4.3).

ACKNOWLEDGEMENT. This work was supported by Junta de Castilla y Leon. 


\section{REFERENCES}

[1] A. R. Aftabizadeh and J. Wiener, Oscillatory and periodic solutions for systems of two first order linear differential equations with piecewise constant argument, Appl. Anal. 26 (1988), no. 4, 327-333. MR 88j:34144. Zbl 634.34051.

[2] A. R. Aftabizadeh, J. Wiener, and J.-M. Xu, Oscillatory and periodic solutions of delay differential equations with piecewise constant argument, Proc. Amer. Math. Soc. 99 (1987), no. 4, 673-679. MR 88b:34099. Zbl 631.34078.

[3] E. Ait Dads and O. Arino, Exponential dichotomy and existence of pseudo almost-periodic solutions of some differential equations, Nonlinear Anal. 27 (1996), no. 4, 369-386. MR 97e:34076. Zbl 855.34055.

[4] E. Ait Dads, K. Ezzinbi, and O. Arino, Pseudo almost periodic solutions for some differential equations in a Banach space, Nonlinear Anal. 28 (1997), no. 7, 1141-1155. MR 98d:34089. Zbl 874.34041.

[5] A. I. Alonso, J. Hong, and R. Obaya, Exponential dichotomy and trichotomy for difference equations, Comput. Math. Appl. 38 (1999), no. 1, 41-49. MR $2000 f: 39001$. Zbl 939.39003.

[6] __ Almost periodic type solutions of differential equations with piecewise constant argument via almost periodic type sequences, Appl. Math. Lett. 13 (2000), no. 2, 131-137. MR 2000m:34162.

[7] A. I. Alonso, J. Hong, and J. Rojo, A class of ergodic solutions of differential equations with piecewise constant arguments, Dynam. Systems Appl. 7 (1998), no. 4, 561-574. MR 99m:34155. Zbl 927.34028.

[8] W. Arendt and C. J. K. Batty, Almost periodic solutions of first- and second-order Cauchy problems, J. Differential Equations 137 (1997), no. 2, 363-383. MR 98g:34099. Zbl 879.34046.

[9] B. Basit and C. Zhang, New almost periodic type functions and solutions of differential equations, Canad. J. Math. 48 (1996), no. 6, 1138-1153. MR 98c:43010. Zbl 880.43009.

[10] J.-M. Belley, G. Fournier, and J. Hayes, Existence of almost periodic weak type solutions for the conservative forced pendulum equation, J. Differential Equations 124 (1996), no. 1, 205-224. MR 96m:34077. Zbl 841.34047.

[11] S. Busenberg and K. L. Cooke, Models of vertically transmitted diseases with sequentialcontinuous dynamics, Nonlinear Phenomena in Mathematical Sciences (Arlington, Tex., 1980), 1982, pp. 179-187. Zbl 512.92018.

[12] L. A. V. Carvalho and K. L. Cooke, A nonlinear equation with piecewise continuous argument, Differential Integral Equations 1 (1988), no. 3, 359-367. MR 89d:34114. Zbl 723.34061.

[13] K. L. Cooke and J. Wiener, Retarded differential equations with piecewise constant delays, J. Math. Anal. Appl. 99 (1984), no. 1, 265-297. MR 85d:34072. Zbl 557.34059.

[14] _ Neutral differential equations with piecewise constant arguments, Boll. Un. Mat. Ital. B (7) 1 (1987), no. 2, 321-346. MR 89e:34107. Zbl 639.34003.

[15]___ A survey of differential equations with piecewise continuous arguments, Delay Differential Equations and Dynamical Systems (Claremont, CA, 1990), Lecture Notes in Math., vol. 1475, Springer, Berlin, 1991, pp. 1-15. MR 92h:34133. Zbl 737.34045.

[16] C. Corduneanu, Almost Periodic Functions, Chelsea, New York, 1989. Zbl 672.42008.

[17] A. M. Fink, Almost Periodic Differential Equations, Lecture Notes in Mathematics, vol. 377, Springer-Verlag, Berlin, 1974. MR 57\#792. Zbl 325.34039.

[18] A. Haraux, Generalized almost periodic solutions and ergodic properties of quasiautonomous dissipative systems, J. Differential Equations 48 (1983), no. 2, 269-279. MR 84g:34109. Zbl 514.34041.

[19] J. Hong, R. Obaya, and A. M. Sanz, Ergodic solutions via ergodic sequences, Nonlinear Anal. 40 (2000), no. 1-8, Ser. A: Theory Methods, 265-277. MR 2001i:37032. Zbl 957.34054 . 
[20] _ Almost periodic type solutions of some differential equations with piecewise constant argument, Nonlinear Anal. 45 (2001), no. 6, Ser. A: Theory Methods, 661-688. CMP 1841201.

[21] G. H. Meisters, On almost periodic solutions of a class of differential equations, Proc. Amer. Math. Soc. 10 (1959), 113-119. MR 21\#2782. Zbl 092.30401.

[22] Z. Opial, Sur les solutions presque-périodiques d'une classe d'équations différentielles, Ann. Polon. Math. 9 (1960), 157-181 (French). MR 23\#A390. Zbl 098.28803.

[23] G. Papaschinopoulos, On asymptotic behavior of the solutions of a class of perturbed differential equations with piecewise constant argument and variable coefficients, J. Math. Anal. Appl. 185 (1994), no. 2, 490-500. MR 95f:34064. Zbl 810.34079.

[24] G. Seifert, Almost periodic solutions for delay-differential equations with infinite delays, J. Differential Equations 41 (1981), no. 3, 416-425. MR 83a:34109. Zbl 461.34053.

[25] S. M. Shah and J. Wiener, Advanced differential equations with piecewise constant argument deviations, Int. J. Math. Math. Sci. 6 (1983), no. 4, 671-703. MR 85e:34077. Zbl 534.34067.

[26] J. Wiener, Differential equations with piecewise constant delays, Trends in Theory and Practice of Nonlinear Differential Equations (Arlington, Tex., 1982), Lecture Notes in Pure and Appl. Math., vol. 90, Dekker, New York, 1984, pp. 547-552. CMP 00741 544. Zbl 531.34059. , Generalized Solutions of Functional-Differential Equations, World Scientific, New Jersey, 1993. MR 94m:34174. Zbl 874.34054.

[28] _ A second-order delay differential equation with multiple periodic solutions, J. Math. Anal. Appl. 229 (1999), no. 2, 659-676. MR 99k:34159. Zbl 920.34060.

[29] J. Wiener and V. Lakshmikantham, Excitability of a second-order delay differential equation, Nonlinear Anal. 38 (1999), no. 1, Ser. B: Real World Appl., 1-11. MR 2000c:34180. Zbl 945.34048.

[30] R. Yuan and J. Hong, Almost periodic solutions of differential equations with piecewise constant argument, Analysis 16 (1996), no. 2, 171-180. MR 97c:34088. Zbl 855.34089.

[31] _ The existence of almost periodic solutions for a class of differential equations with piecewise constant argument, Nonlinear Anal. 28 (1997), no. 8, 1439-1450. MR 98e:34135. Zbl 869.34038.

[32] C. Zhang, Pseudo almost-periodic functions and their applications, Ph.D. thesis, University of Western, Ontario, 1992.

[33] _ Pseudo-almost-periodic solutions of some differential equations, J. Math. Anal. Appl. 181 (1994), no. 1, 62-76. MR 95c:34081. Zbl 796.34029.

Ana Isabel Alonso: Departamento de Matemática Aplicada a la Ingeniería, Paseo DEL CAUCE S/N. 47011, UNIVERSIDAD DE VALLADOLID, SPAIN

E-mail address: anaa1o@wmatem. eis.uva.es

JiALIN Hong: INSTITUTE OF COMPUTATIONAL MATHEMATICS AND SCIENTIFIC/ENGINEERING COMPUTING, CHINESE ACADEMY OF SCIENCES, BEIJING 100080, ChinA

E-mail address: hj1@1sec.cc.ac.cn 


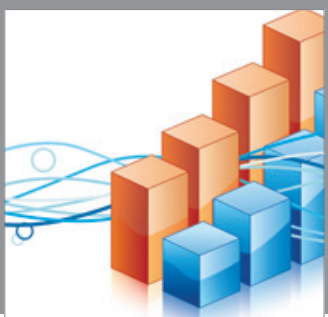

Advances in

Operations Research

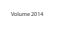

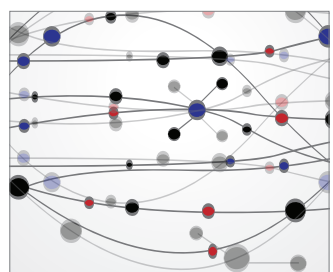

\section{The Scientific} World Journal
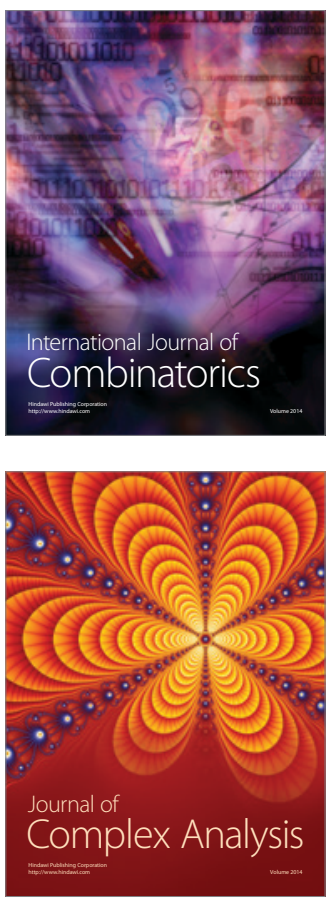

International Journal of

Mathematics and

Mathematical

Sciences
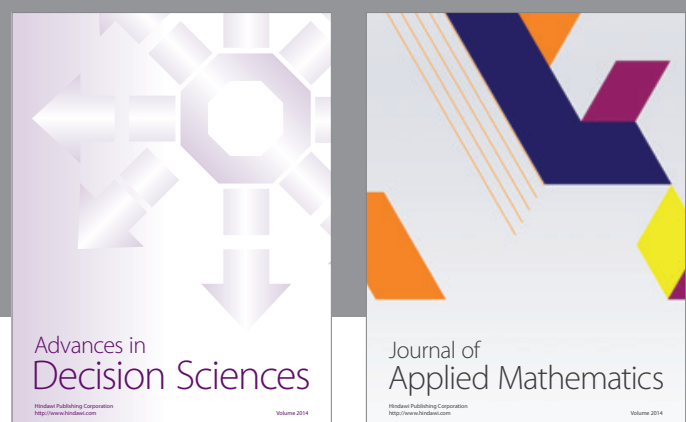

Journal of

Applied Mathematics
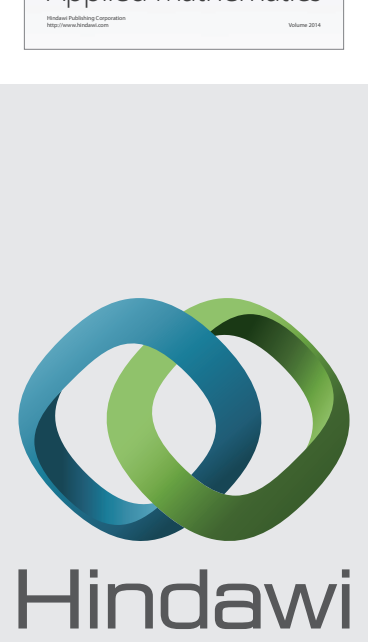

Submit your manuscripts at http://www.hindawi.com
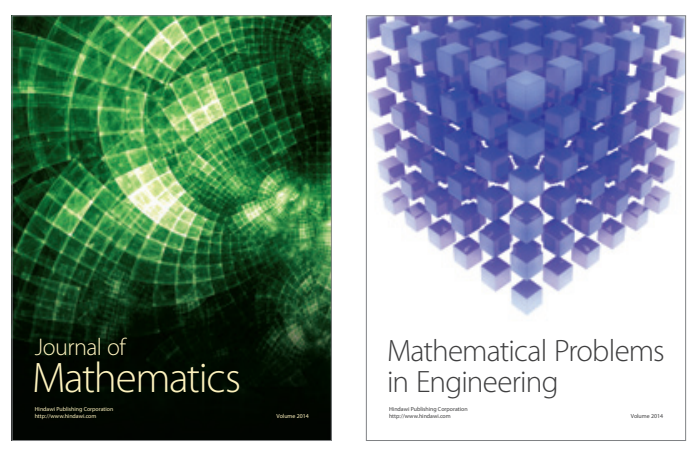

Mathematical Problems in Engineering
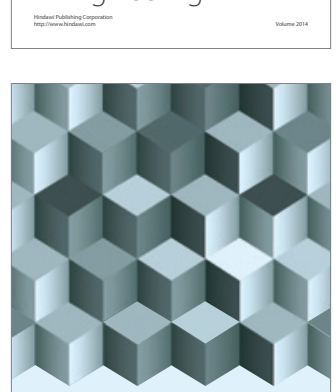

Journal of

Function Spaces
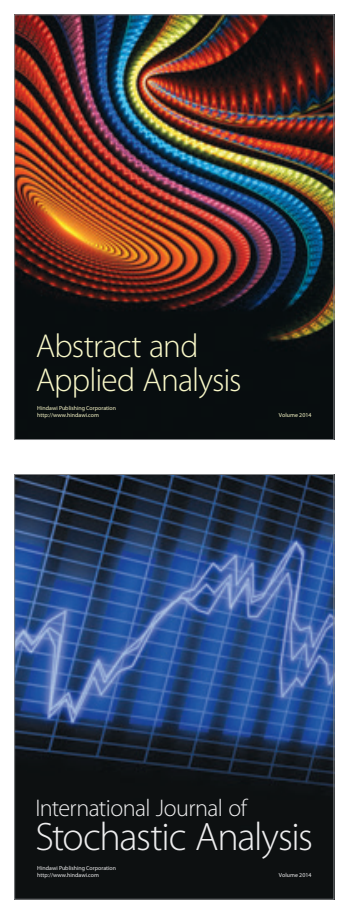

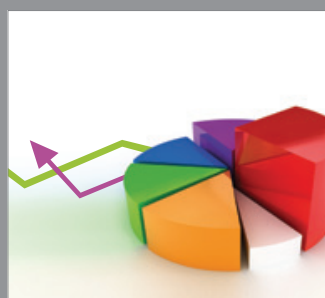

ournal of

Probability and Statistics

Promensencen
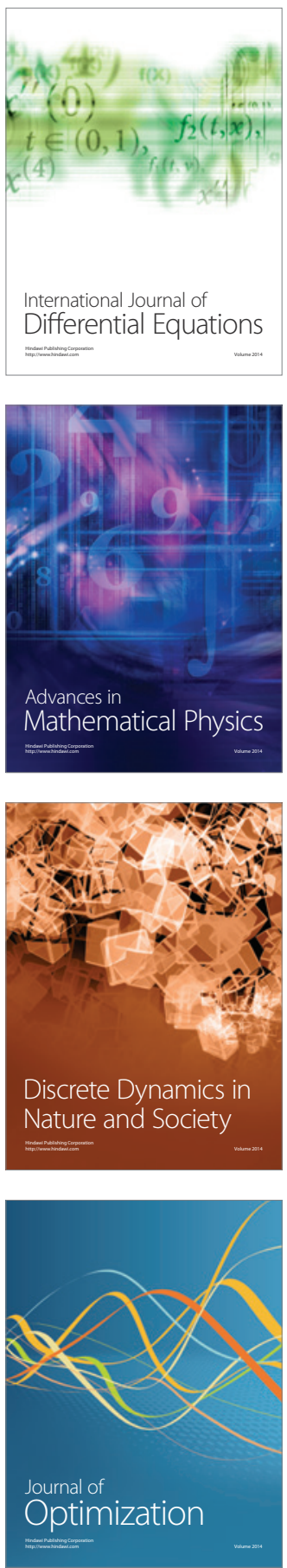